\title{
Hommage au Professeur Jean-Pierre Delagoutte
}

\section{Tribute to Professor Jean-Pierre Delagoutte}

\section{P.H. Benamou}

(C) Lavoisier SAS 2015

Le Professeur Jean Pierre Delagoutte a été membre de la Société Française de Médecine et Chirurgie du Pied (SFMCP), depuis 1974. Il en a assuré la Présidence de 1980 à 1982.

Dans son allocution de départ, il a cité le poème d'Alfred de Vigny, « La mort du loup » :

«Fais énergiquement ta longue et lourde tâche

Dans la voie où le Sort a voulu t'appeler,

Puis après, comme moi, souffre et meurs sans parler. »

C'est exactement ce qu'il a fait et vécu jusqu'au bout. Sa maladie, il en parlait peu. Je ne l'ai su que tardivement.

Il était très discret sur son parcours professionnel et ses activités scientifiques... même vis-à-vis de son entourage familial.

Je ne parlerai que de ses activités au sein de la Société française de médecine et chirurgie du pied. Les Professeurs Coudane et Mainard se sont chargés de son parcours hospitalier dans un "In Memoriam » qu'ils ont rédigé pour la Revue de Chirurgie Orthopédique et pour la revue Médecine et Chirurgie du Pied.

La Présidence de Jean-Pierre Delagoutte à la SFMCP, a été marquée par les rapports difficiles avec le Collège International de Podologie (CIP) et l'indépendance de la SFMCP vis-à-vis du CIP.

Il a été à l'origine de la publication des Actes des Congrès de la SFMCP dans la revue Cinésiologie avant la création de la revue Médecine et Chirurgie du Pied, par le Docteur Simon Braun en 1984.
Le comité de lecture est créé en 1986 et il en a assuré la coordination jus-

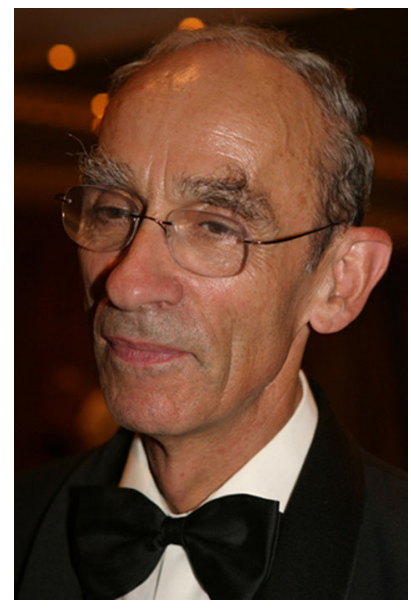
qu'à son décès.

J'ai eu le privilège de travailler avec lui, comme Directeur de rédaction de la revue depuis 1998 et nos rapports ont toujours été empreints de respect et d'amitié.

Nous avons connu différents éditeurs :

- l'Expansion Scientifique de 1984 à 1999 ;

- Elsevier en 2000 ;

- Springer-Verlag de 2001 à 2014 ;

- Lavoisier depuis 2015.

Sa discrétion était telle que j'ai rarement pu connaître les noms des « relecteurs » de tel ou tel article.

Je le rencontrais fréquemment lors de Journées annuelles de rhumatologie de l'hôpital Cochin, car il était persuadé que médecins et chirurgiens étaient indispensables à l'étude du pied.

À ses enfants Véronique, Anne-Sophie, Emmanuelle et Bénédicte, ainsi qu'à ses petits enfants Basile, Camille et Paul, et à toute la famille, je présente mes condoléances au nom de la Société française de médecine et chirurgie du pied.

Docteur Paul-Hubert Benamou

Directeur de la Rédaction

P.H. Benamou ( $₫)$

56, avenue du Président Kennedy, F-92160 Antony

e-mail : benamoup@club-internet.fr 